Leifson, E. \& Hugh, R. (1954). J. gen. Microbiol. 10, 68-70

\title{
A New Type of Polar Monotrichous Flagellation
}

\author{
By E. LEIFSON AND R. HUGH \\ Department of Microbiology, Stritch School of Medicine and Graduate School, \\ Loyola University, Chicago 12, Illinois, U.S.A.
}

SUMMARY : An organism with a new type of polar monotrichous flagella is described. The flagella have a very short wavelength which is very characteristic. The organism liquefies gelatin and oxidizes ethanol to acid. All other common differential tests were negative. The name Pseudomonas diminuta n.sp. is suggested.

The curvature of bacterial flagella is quite constant and sufficiently characteristic to be of considerable taxonomic importance. The usual wavelength of the flagella of such polar monotrichous bacteria as Pseudomonas aeruginosa and Vibrio comma is in the neighbourhood of $2 \mu$. In a large collection of cultures of Gram-negative bacteria submitted to the authors for flagellar study by Robert Keller of the Museum of Natural History of Philadelphia were found three cultures which showed very unusual monotrichous flagella, having a wavelength of only slightly over $0 \cdot 6 \mu$. The cultures originated from fresh-water streams. Morphologically and physiologically the three cultures were identical and one description will apply to all.

\section{MORPHOLOGY OF ORGANISM}

The organism is a small Gram-negative rod averaging 0.5 by $1.0 \mu$ in the usual peptone media. Capsule or spore formation was not demonstrable. It is actively motile with predominantly a single polar flagellum. A flagellum at each pole is fairly common in young cultures, and occasionally two flagella may be found on one pole. The flagella have two unusual characteristics: (1) Many of the individuals show the flagellum originating from the periphery of the pole rather than from the centre of the pole, the latter being the more usual origin with monotrichous bacteria. (2) The flagellar curvature is most unusual with an average wavelength of only $0.62 \mu$, and an average wave amplitude of $0.32 \mu$. The standard deviation of the wavelengths from the mean, based on measurements of twenty-five flagella, is $0 \cdot 12 \mu$ and the standard error of the mean wavelength is $0.024 \mu$. In Fig. 1 is illustrated the flagellar morphology of the organism. The insert in the lower right corner shows the flagellar morphology of a typical strain of Pseudomonas aeruginosa.

The wavelength of the flagella of the strain of Ps. aeruginosa pictured is most common with monotrichous types of bacteria and averages slightly over $2 \mu$. This statement is based on the study of over 100 strains, representing many, but mainly unclassified, species. Among the named species of Pseudomonas studied may be mentioned Ps. stutzeri, Ps. fuorescens, Ps. saccharophila, Ps. mucidolens, and the polar multitrichous Ps. marginalis. The flagella were stained according to the method of Leifson (1951). 


\section{GROWTH CHARACTERISTICS AND PHYSIOLOGY}

The organism grows very readily in a simple peptone solution. The optimum $\mathrm{pH}$ is around $\mathbf{7 \cdot 0}$ and the optimum temperature around $35^{\circ}$. Liquid media become densely turbid with only a slight pellicle. On the surface of agar media the growth is smooth, raised, and white to creamy in colour. No distinct pigments are produced. Individual colonies are raised, smooth and cream coloured. The original cultures showed two types of colonies: (1) uniformly opaque, and (2) somewhat translucent with an opaque centre and opaque periphery. Isolates from the two types of colonies showed no differences morphologically or physiologically.

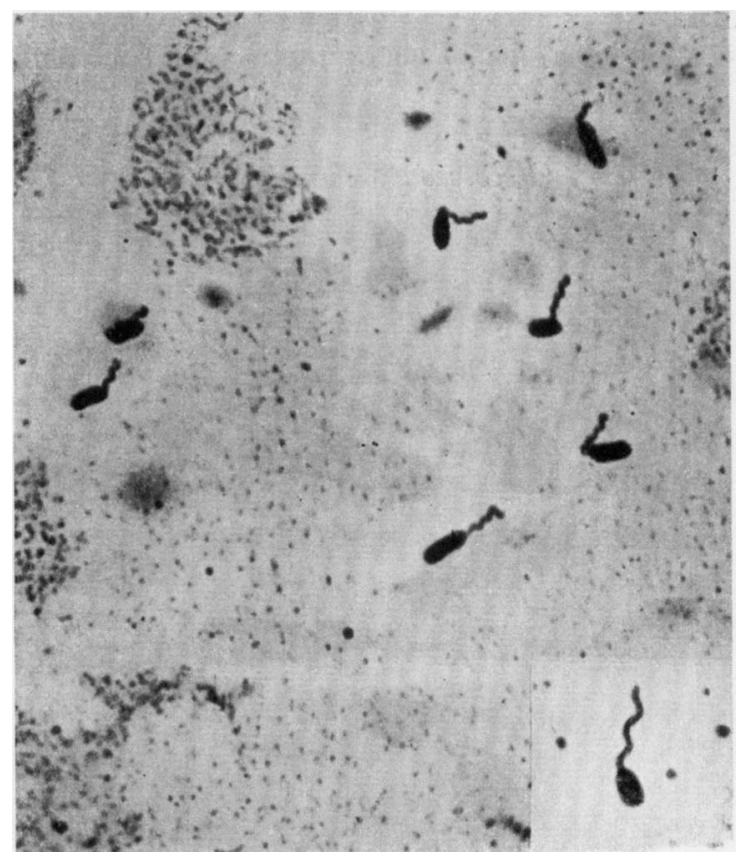

Fig. 1. Typical flagellation of Ps. diminuta n.sp. Insert in lower right corner shows the typical flagellation of Ps. aeruginosa. Note the curvature of the flagella on the two types of organisms. Leifson flagella stain. Photomicrographs of 2000 magnification.

Detectable acid was not produced from any of the carbohydrates tested including glucose, sucrose, lactose, maltose, arabinose, xylose and galactose. The following tests were negative: indole, MR, VP, urea, $\mathrm{H}_{2} \mathrm{~S}$, nitrate and citrate. Litmus milk became blue and translucent. Subsurface colonies in blood agar showed no definite haemolysis. Gelatin was liquefied in about 6 days at $30^{\circ}$. Ethanol was oxidized to acid very promptly and in concentration up to and including $5 \%(\mathrm{v} / \mathrm{v})$. This latter reaction appears somewhat unusual for an organism which has no effect on carbohydrates. The tests with carbohydrates and ethanol were made using the basal medium described by Hugh \& Leifson (1953). 


\section{DISCUSSION}

On the bases of flagellar arrangement and physiology the organism seems, for the present, best fitted into the genus Pseudomonas. The shape of the flagella is so unusual and characteristic that a new species designation seems appropriate. The name $P$ s. diminuta n.sp. is proposed for the organism.

A culture has been deposited in the National Collection of Type Cultures (NCTC 8545).

\section{REFERENCES}

HUGh, R. \& Lerfson, E. (1953). The taxonomic significance of fermentative versus oxidative metabolism of carbohydrates by various Gram-negative bacteria. J. Bact. 66, 24.

Lerfson, E. (1951). Staining, shape, and arrangement of bacterial flagella. J. Bact. 62, 37\%.

(Received 16 June 1953) 